den Schluss der Sitzung. Es wurde beschlossen eine anderthalbtägige Fahrt nach dem Ober-Spreewald, beginnend am Mittage des ersten oder zweiten Sonnabend nach Pfingsten, zu unternehmen. Zur Vorbereitung derselben werden die Herren $\mathrm{Cab}$ a $\mathrm{n}$ is und $B$ tinger gewählt.

Möbius.

Vorsitzender.
Matschie. Schriftführer.
$\mathrm{Cab}$ a nis. Gen.-Secr.

\title{
Bericht über die September-Sitzung 1892.
}

Ausgegeben am 24. September 1892.

Verhandelt Berlin, Montag, den 5. September 1892 , Abends $\delta$ Uhr, im Sitzungslocale, Bibliothekzimmer des Architekten-Vereinshauses, Wilhelmstr. 92 II.

Anwesend die Herren: Cabanis, Reichenow, Hartwig, Frenzel, Thiele, Matschie, Bünger, Pascal, Kühne, Rörig, Grunack, Hocke und Nauwerck.

Als Gast: Herr Dr. Lange (Berlin).

Vorsitzender: Herr Cabanis. Schriftf.: Herr Matschie.

Als Mitglieder sind der Gesellschaft im Laufe der Sommerferien beigetreten die Herren: Ingenieur C. Pallisch, Herausgeber der Mittheilungen des ornithologischen Vereines in Wien "Die Schwalbe", in Erlach bei Wiener Neustadt, Niederösterreich, E. Schreiner, Procurist, Berlin, Udo Lehmann in Neudamm, Professor Dr. Frenzel in Friedrichshagen bei Berlin, Capt. Shelley in London, Ladislar Kenessey von Kenessey in Stuhlweissenburg, Ungarn.

Herr $\mathrm{Cabanis} \mathrm{legt} \mathrm{die} \mathrm{seit} \mathrm{der} \mathrm{Mai-Sitzung} \mathrm{erschienenen}$ Nummern der deutschen ornithologischen Zeitschriften vor und hebt aus dem Inhalte derselben einige interessante Mittheilungen hervor.

Von H. Nehrling's: „Die Nord-Amerikanische Vogelwelt" ist das 13. Heft als Schluss-Lieferung nunmehr erschienen. Das W.erk beschränkt sich auf Sing-, Schrei- und Klettervögel und führt von jeder Gattung die am meisten in die Augen fallenden und wohl auch am meisten studirten Arten in Wort und Bild vor. Herrn Nehrling ist es vornehmlich darum zu thun gewesen, in der Jugend 
den Sinn fur Wald, Flur und Vogelsang zu erwecken. Seine Schilderungen sind klar und lebhaft, und die Abbildungen, von G. Mützel, Goering und Ridgway zum grossen Theil vorzüglich.

J. A. Allen beschreibt eine neue Gallinula: Porphyriornis comeri gen. et sp. nov. von den Gongh- Inseln, 200 Meilen sudwestlich vom Cap der guten Hoffnung (Am. Mus. Nat. Hist. IV. 1. p. 57, 58. 1892).

H. E. Dresser giebt eine Uebersicht über die nächsten Verwandten von Lanius lahtora im "Ibis". 1892, April.

E. Hartert bringt in der April-Nummer des "Ibis" Bemerkungen über einige schwierige Fragen, welche die Caprimulgidae betreffen und bildet den seltenen Caprimulgus eximius Temm. ab.

G. Hartlaub beschreibt (Ibis, Juli 1892) eine Hyliota nehrkorni von Accra; die beigegebene Abbildung giebt den Farbenton der Oberseite nicht richtig wieder; die Art ist oben schwarz, mit geringem stahlblauen Schimmer.

Hartla ab giebt in den Abh. d. naturw. Ver. z. Bremen XII. Bd. Heft 2 einen Beitrag zur Ornithologie Chinas. Er behandelt 45 Arten von Tientsin, Nord-China, 23 Arten von Shanghai, 9 Arten von Ningkuofu am Jang-tse, 28 Arten von Formosa, 92 Arten von Hainan und 11 Arten von Pakhoi am Golf von Tonkin.

E. C. F. Rzehak hat taber die Avifauna Oesterreich-Schlesiens einige kleinere interessante Arbeiten theils in der "Schwalbe“, theils in den Mitth. d. K.-K. mäbr.-schles. Gesellschaft für Ackerbau, Natur- und Landeskunde veröffentlicht; die Raubvögel Schlesiens, System. Verz. d. bisher in Oestr.-Schlesien beobachteten Vögel und Beiträge zur Kenntniss der heimischen Vogelwelt.

Herr R e i c hen ow legt folgende neu erschienene Arbeiten vor:

A. Suchetet, Les oiseaux hybrides, rencontrés à l'état sauvage. 3. Partie. Les Passereanx; Mém. Soc. Zool. France T. V. S. 179-451. - Den mit unermtidlicher Ausdauer fortgesetzten Bemulungen des Verfassers ist es gelungen, ein ungemein reiches Material zu sammeln. Gegen 100 Bastardformen aus den Gruppen der Singvögel, Raken und Spechte sind in dem vorliegenden dritten Theil der Arbeit besprochen. Für die Mehrzahl der Formen sind nur einzelne, für manche aber eine grössere Anzahl von Fällen nachgewiesen. So führt Verf. 7 Beispiele der Hybridation von Hirundo urbica und rustica auf. Am Schlusse wird noch zweier 
Curiosa gedacht, deren thatsächliches Vorkommniss dem Verf. von Correspondenten versichert wurde, nämlich der Hybridation von Saxicola rubicola $\times$ Carduelis elegans und Ruticilla sp.? $\times$ Carduelis. Beide gebören offenbar in das Reich der Fabeln. Unter den authentischen Fällen der Hybridation einander ferner stehender Arten dürfte der von Emberiza citrinella $\times$ Cynchramus schoeniclus einer der interessantesten sein.

J. V. Barboza du Bocage, Aves do Sertão de Benguella; Jorn. Sc. Math. Phys. Nat. Lisboa (2.) VII. S. 157-172. - Bespricht eine Sammlung des bekannten Reisenden Anchieta von Quindumbo, Quibula und Cahata in Benguella, 71 Arten, darunter Buteo auguralis, Telephonus ussheri [ein auffallend stidlicher Verbreitungspunkt dieser Art], hingegen die stidliche Thamnolaea shelleyi.

Derselbe, Aves de Dahomé: ebenda S. 185-187. - 16 Arten, gesammelt ron $\mathrm{F}$. Newton, werden aus dem bisher noch wenig erforschten Gebiet aufgezählt.

G. H artla ub, On a new Species of Flycatcher of the Genus Hyliota: Ibis 1892 S. 373-374. - Hyliota nehrkorni n. sp. von Accra beschrieben. Es sind: nunmehr 5 Arten der Gattung bekannt.

Derselbe, Ein Beitrag zur Ornithologie Chinas: Abh. naturw. Ver. Bremen 12. S. 295-335. - Behandelt drei Sammlungen, welche im Jahre 1891 an das Bremer Museum gelangten. Die erste wurde von Herrn B. Schmacker auf Formosa zusammengebracht, die zweite von Herrn A. Schomburg vorzinglich auf Hainan; die dritte stammt vom Norden Chinas aus dem Gebiete von Tientsin, von Herrn A. Walte gesammelt. Dieselben repräsentiren 186 Arten. U. a. wird die in Sammlungen noch sehr seltene Larvivora sibilans Swinh, für Hainan nachgewiesen. In der Einleitung schildert Verf. die Topographie der genannten Sammelgebiete und giebt eine Uebersicht der wichtigsten einschlägigen Arbeiten.

J. Buttik ofer, On a Collection of Birds from the Islands of Flores, Sumba and Rotti: Notes Leyden Mus. 14. S. 193-206. - Bespricht eine von Dr. H. ten Kate auf den genannten Inseln der Timor-Gruppe zusammengebrachte Sammlung. Von Flores wird Acanthiza tenkatei n. sp. beschrieben. Von der noch wenig bekannten Insel Sumba fuhrt Verf. 32 Arten auf, welche deren faunistische. Uebereinstimmung mit Flores beweisen, darunter 
Dicaeum wilhelminae n. sp.; eingehender werden die Kennzeichen von Munia nisoria (Tem.) und deren verwandte Formen besprochen. Von der Insel Rotti, einem kleinen Eiland am Westende von Timor, sind 5 Arten aufgefthhrt, darunter Rhipidura tenkatei n. sp.

A. B. Meyer, The Birds of Sumba: ebenda S. 265-268. - Verf. weist darauf hin, dass Dr. Riedel bereits vor mehr als einem Jahrzehnt auf der Insel Sumba ornithologisch gesammelt hat [rergl. . vorgehende Arbeit], welche Collection vom Verf. in den Verh. Zool. Bot. Ges. Wien 1881 S. 759-767 beschrieben worden ist. Von den daselbst aufgeführten 40 Arten decken sich nur 8 mit den von ten Kate auf Sumba gefundenen Species, so dass bis jetzt 64 Arten von der Insel bekannt sind. Die subspecifische Sonderung des 1. c. beschriebenen Tanygnathus megalorhynchus sumbensis hält Verf. gegentiber der abweichenden Ansicht Salvadori's aufrecht und unterscheidet auch den fruher als Geoffroyus jukesii aufgefuhrten Papagei als selbständige Art: Geoffroyus tjindanae n. sp. An Stelle des in der angezogenen Arbeit S. 767 irrthiumlich aufgefuhrten Herodias nigripes ist zu setzen : Bubulcus coromandus. Unter den 64 bekannten Sumba-Arten befinden sich nunmehr 5 der Insel eigenthlimliche: Ninox rudolfi, Grancalus sumbensis, Dicaeum wilhelminae, Tanygnathus sumbensis und Geoffroyus tïindanae.

J. Buttik ofer, On the Collections of Birds sent by the late A. T. Demery from the Sulymah River (W.-Afrika): ebenda S. $19-30$. - y6 Arten, von welchen 10 bisher in Liberia nicht gefunden sind. Die specifische Verschiedenheit von Malimbus rubricollis und $M$. bartletti bezweifelt Verf. Das $q$ von $\boldsymbol{M}$. malimbicus (Daud.) unterscheidet sich rom of nur durch Fehlen der Haube. Besonders interessant ist der Nachweis ron Coliopasser concolor (Cass.) im Gebiete (vergl. J. O. 1891 S. 46).

Th. Pleske, die ornithologische Ausbeute der Expedition der Gebrüder G. und M. Grum-Grzimailo nach Centralasien (1889-90); Bull. Ac. Imp. Sc. St. Petersbourg T. 13. S. 273-301. - Die Expedition, welche namentlich den östlichen Tjan-schan, das Gebirgsland Bei-schan, das Njan-schan-Gebirge sowie das Gebirgslaud Amdo and Kuku-nor genauer erforschte, ergänzt in vorzüglicher. Weise die Przewalski'schen Forschungen. Die Erforschung des Njan-schan hat das interessante Ergebniss geliefert, dass die Nordgrenze vieler central-chinesischer Arten, die bisher nur bis Amdo nachgewiesen waren, bedeutend nördlicher an den 
Nordabhang des Njan-schan versetzt werden muss. Die gesammelten 1048 Vogelbälge repräsentiren 191 Arten, darunter Accipiter virgatus, Falco babylonicus, Erythropus amurensis, Loxia curvirostra himalayana, Jugendform von Crossoptilon auritum und Dunenjunge von Ibidorhynchus, ferner Eier von Chloris sinica, Carpodacus pulcherrimus, Parus superciliosus, Herbivocula affinis, Perdix sifanica und Phas. strauchi. Der von Przewalski beschriebene Phas. satscheuensis wird im Vergleich zu Ph. torquatus eingehender beschrieben.

Derselbe, Uebersicht der Gattung Regulus Cur.: ebenda S. 303-307. - 14 Arten werden unterschieden, darunter Regulus tristis n. sp. von Transkaspien und Turkestan. Die Kennzeichen der einzelnen Arten sind in Form eines Schlüssels übersichtlich dargestellt.

V. จ. Tschusi-Schmidhoffen, Am Velenczeer- und Kleinen Balatonsee: Ber. II. intern. orn. Congress Budapest. Schilderung der im Anschluss an den genannten Congress unternommenen Excursion. Lusciniola melanopogon wurde vielfach nistend gefunden, am Balaton wurden namentlich Colonien von Ardea alba und ralloides sowie Nycticorax griseus besucht.

Herr $B$ ü ng er bespricht einen in den Jahresberichten der ornitholog. Gesellschaft in Basel veröffentlichten Aufsatz des Herrn Professor Dr. F. Zschokke über Wandertrieb und Wanderungen der Vögel.

Herr Frenzel hält einen längeren Vortrag über die Vogelwelt Argentiniens, in welchem er nach einer Beschreibung der in der Umgebung von Córdoba zusammentreffenden 3 topographischen Regionen, der Pampas, des Monte, der Sierra, eine Uebersicht der jedem dieser Gebiete angehörigen Vogelformen giebt.

Herr $\mathrm{Cab}$ a is legt einige sebr interessante ornithologische Objekte vor, welche unser Mitglied Alessi in Monastir; Tunis, eingesendet hat. Es ist ein Exemplar der für Tunis seltenen, daselbst noch nicht nachgewiesenen Pterocles coronata, ein Exemplar der Otocorys bilopha und Eier von Alaemon margaritae.

Herr Reichenow legt die nachfolgenden neuen Arten vor:

Laniarius gladiator Rchw. n sp.

Capite et cervice, mento et gulae parte superiore cinereis, his olivaceo-lavatis; corpore toto reliquo, cauda et alis olivaceoviridibus; rectricibus intus tenuiter flavescente limbatis; remigibus nigrofuscis extra olivaceo-viridi-, intus dilute flavo-marginatis; 


\section{Allgem. Deutsche Ornitholog. Gesellschaft zu Berlin:}

rostro nigro; pedibus plumbeis; iride cinerea. L. t. 270 , a. im. 120 , c. 120 , r. 30 , t. $36 \mathrm{~mm}$. Hab. Buea (Kamerun) $1000-1500 \mathrm{~m}$ (Preuss. c.).

Symplectes preussi Rchw. n. sp.

Pileo aureo-brunneo; cervice, dorso et uropygio citrinis; capitis et colli lateribus, gula, scapularibus, alis, cauda et supracandalibus nigris, his macula apicali citrina notatis, remigibus interioribus limbo apicali flavido; gastraeo reliquo citrino; subalaribus albis. L. t. 140 , a. im. 85 , c. 45, r. 17, t. $20 \mathrm{~mm}$. Victoria (Kamerun) (Preuss c.).

Psalidoprocne chalybea Rchw. n. sp.

P. obscurae simillima sed nitore chalybeo olivascente. Nigra, nitore viridi-Malybeo, dorso olivascente viridi- splendente, gastraeo obscuriore; subalaribus cinereo-brunneis; cauda furcata. L. t. 160 , a. im. 98 , c. 95 , r. $4-5$, t. $9 \mathrm{~mm}$. Hab. Victoria (Preuss c.).

Herr Hartwi ig theilt mit, dass er von Madeira Ei und Balg (ठ) von Sylvia conspicillata Marm. in diesem Frubjahr erhalten habe, wodurch das Brutvorkommen dieser Art auf Madeira bewiesen werde. Ferner sei ihm ein $\delta$ im Sommerkleide von Charadrizes squatarola (L.) aus Madeira im August 1892 zugegangen. Die Gesammtzahl der in Madeira beobachteten Vögel erhöht sich dadurch auf 118 Species.

Herr Hocke legt das Nest eines Grün-Hänflings (chloris) mit lauter Doppeleiern vor, in welchem ein Kukuksei gefunden wurde. Ferner weist der Redner darauf hin, dass in diesem heissen Sommer von ihm Ohreulen ' $\delta$ und $q$ mit ganz ausgebreiteten Flügeln dasitzend gefunden seien.

Schluss der Sitzung

$$
\begin{aligned}
\text { Matschie, } & \text { Cabanis, } \\
\text { Schriftführer. } & \text { Gen.-Secr. }
\end{aligned}
$$

Wegen der vom 1.-4. $0 \mathrm{c}$ t o b e r hier in Berlin abzuhaltenden Jahresversammlung der Gesellschaft fällt die Monatssitzung im October aus. 